\title{
THE EXTREME PROJECTIONS OF THE REGULAR SIMPLEX
}

\author{
P. FILLIMAN
}

\begin{abstract}
The largest and smallest projections of the regular $n$-dimensional simplex into a $k$-dimensional subspace are determined for certain values of $n$ and $k$. These results suggest that the smallest $k$-dimensional projection and the largest $(n-k)$-dimensional projection occur in orthogonal subspaces of $R^{n}$.
\end{abstract}

\section{INTRODUCTION}

Let $T^{n}$ be the regular $n$-dimensional simplex with edge length $\sqrt{2}$, and let $P=\Pi\left(T^{n}: L\right)$ be the orthogonal projection of $T^{n}$ into a $k$-dimensional subspace $L$ of $R^{n}$. Our goal is to find the projections of $T^{n}$ with the maximum and minimum $k$-volume.

A striking pattern has emerged in the results below, which leads to the following conjectures: Let $T_{n}^{k}=\Pi\left(T^{n}: L_{0}\right)$ be a $k$-dimensional simplex with evenly distributed weights, where the weights of a vertex is the number of vertices of $T^{n}$ which project onto it.

Conjecture A. The smallest $k$-dimensional projection of $T^{n}$ is $T_{n}^{k}$.

Conjecture B. The largest $(n-k)$-dimensional projection of $T^{n}$ is

$$
\bar{T}_{n}^{k}=\Pi\left(T^{n}: L_{0}^{\perp}\right) \text {. }
$$

If true, these conjectures would imply that the largest and smallest projections of $T^{n}$ occur in orthogonal subspaces, and are therefore Gale transforms of each other (see $[12, \S 3.1])$. This can be contrasted with the case of the regular $n$-cube, in which such projections have the same volume (see [2, 3.7]).

The main tool we shall use in the exterior algebra method of [6], which is summarized in $\S 1$. $\S 2$ deals with minimal projections, and $\S 3$ with maximal projections, where results are given when $k$ or $n-k$ is small (see also [7] and [10]). The bulk of $\S 3$ concerns the case $k=n-2$. The determination of the maximal projection of a fixed combinatorial type is reduced to a rather unusual counting problem on the corresponding 2-dimensional Gale diagram. The tables

Received by the editors February 16, 1988.

1980 Mathematics Subject Classification (1985 Revision). Primary 52A25; Secondary 51M25. 
at the end of the paper give bounds for the volumes of the projections whose diagrams are pictured in $[8, \mathrm{p} .112]$.

\section{Preliminaries}

The exterior algebra $\Lambda_{k} R^{n}$ is a real vector space of dimension $\left(\begin{array}{l}n \\ k\end{array}\right)$, and its elements are called " $k$-vectors." If $\left\{e_{1}, \ldots, e_{n}\right\}$ is the standard basis of $R^{n}$, then the $k$-vectors

$$
\begin{gathered}
e_{\lambda}=e_{\lambda_{1}} \wedge \cdots \wedge e_{\lambda_{k}} \\
\left.\lambda \in \Lambda(n, k)=\left\{\lambda_{1}, \ldots, \lambda_{k}\right) \mid 1 \leq \lambda_{1}<\cdots<\lambda_{k} \leq n\right\},
\end{gathered}
$$

form an orthonormal basis of $\Lambda_{k} R^{n}$. A $k$-vector $\xi \in \Lambda_{k} R^{n}$ is said to be "simple" if $\xi=x_{1} \wedge \cdots \wedge x_{n}, x_{i} \in R^{n}$. If $\xi \neq 0$, we let $L(\xi)$ denote the $k$-plane $\operatorname{lin}\left\{x_{1}, \ldots, x_{k}\right\}$, and we denote the collection of all $k$-dimensional subspaces of $R^{n}$ by $G(k, n)$. In case $x_{1}, \ldots, x_{n}$ is an orthonormal basis of $L(\xi)$, then the usual Euclidean norm of $\xi$ is $|\xi|=1$. This gives an embedding of the Grassmannian $\widetilde{G}(k, n)$ of oriented $k$-planes in $R^{n}$ as a submanifold of the unit sphere in $\Lambda_{k} R^{n}$.

The $k$-dimensional volume $V\left(T^{n}: L\right)$ of the projection of $T^{n}$ into a $k$-plane $L$ defines a real-valued function on $\widetilde{G}(k, n)$. The main reason that exterior algebra is so useful for the projection problem is that $V(T: \cdot)$ is locally linear on $\widetilde{G}(k, n)$. That is, if $P=\Pi\left(T^{n}: L\right)$ and $\xi \in \widetilde{G}(k, n)$ is an orientation of $L$, then there exists a $k$-vector $\Phi \in \Lambda_{k} R^{n}$ such that

$$
V(P)=\langle\Phi, \xi\rangle
$$

(see [6, Theorem 1]). This formula holds in a region $\chi(\Phi) \subset \widetilde{G}(k, n)$ in which the projections have the same combinatorial type as $P$. In finding extreme projections, we generally work with each region $\chi(\Phi)$ separately.

We mention one more important point. It will be convenient for calculations to let $T^{n}=\operatorname{conv}\left\{e_{1}, \ldots, e_{n+1}\right\} \subset R^{n+1}$. If $H$ is the $n$-dimensional subspace of $R^{n+1}$ parallel to aff $T^{n}$, then the inclusion $i: H \rightarrow R^{n}$ induces an inclusion $\hat{i}: \widetilde{G}(k, n) \rightarrow \widetilde{G}(k, n+1)$. We shall assume all subspaces belong to $H$, and that their orientations belong to $\hat{i}(\widetilde{G}(k, n))$. This restriction is not so important for maximal projections since [6, Proposition 2] shows that the largest projection must lie in $H$. However, it is crucial for minimal projections since we can choose $L \in G(k, n+1), L \nsubseteq H$, with $V\left(T^{n}: L\right)$ as small as desired.

\section{Minimal PROJECTIONS}

From the remarks above, a "projection form" $\Phi \in \Lambda_{k} R^{n}$ has the property that

$$
V(P)=\langle\Phi, \xi\rangle
$$

where $P=\Pi\left(T^{n}: \xi\right)$ and $\xi \in \chi(\Phi) \subset \widetilde{G}(k, n)$. The key observation concerning 
minimal projections is

Proposition 1. If an arc of a great circle lies in $\chi(\Phi)$ and contains $\xi$ in its interior, then $V\left(T^{n}: \cdot\right)$ could not have a local minimum at $\xi$.

Since the right-hand side of (2.1) is the usual inner-product of $\Lambda_{k} R^{n}$, the proof of this lemma is a simple exercise in 3-dimensional linear algebra.

In order to apply Proposition 1, we shall need a description of great circles on $\widetilde{G}(k, n)$ (see [9, Vol. II, pp. 314-315]).

Proposition 2. The following are equivalent for $\xi, \xi^{\prime} \in \widetilde{G}(k, n)$ with $\left\langle\xi, \xi^{\prime}\right\rangle=0$ :

(a) $\xi$ and $\xi^{\prime}$ can be connected by a great circle in $\widetilde{G}(k, n)$,

(b) $\operatorname{dim}\left(L(\xi) \cap L\left(\xi^{\prime}\right)\right)=k-1$, and

(c) $\xi \in T_{\xi}^{\prime} \widetilde{G}(k, n)$ and $\xi^{\prime} \in T_{\xi} \widetilde{G}(k, n)$,

where $T_{\xi} \widetilde{G}(k, n)$ is the tangent space to $\widetilde{G}(k, n)$ at $\xi$. Also, given an arbitrary tangent vector $\xi^{\prime} \in T_{\xi} \widetilde{G}(k, n)$, (a) holds if and only if $\xi^{\prime}$ is simple.

Proposition 2 implies that the great circles through $\xi$ are in one-to-one correspondence with the simple tangent vectors at $\xi$. If $\xi^{\prime} \in T_{\xi} \widetilde{G}(k, n)$ is simple, then from (b) we may write

$$
\left.\xi^{\prime}=t \wedge(x\rfloor \xi\right)
$$

where $x \in L(\xi), t \in L(\xi)^{\perp}$ and “」” is interior multiplication.

The propositions above can be used to show that certain regions $\chi(\Phi)$ cannot contain a point $\xi$ at which $V\left(T^{n}: \cdot\right)$ is minimized. Given $\mu \in \Lambda(n+1, k+1)$, we let $F_{\mu}=\operatorname{conv}\left\{e_{\mu_{1}}, \ldots, e_{\mu_{k+1}}\right\}$ and let $f_{\mu}=\left(e_{\mu_{2}}-e_{\mu_{1}}\right) \wedge \cdots \wedge\left(e_{\mu_{k+1}}-e_{\mu_{1}}\right)$. Then

$$
\begin{gathered}
\chi(\Phi)=\left\{\eta \in \widetilde{G}(k, n) \mid \operatorname{sign}\left(\left\langle\eta, f_{\mu}\right\rangle\right)=\operatorname{sign}\left(\left\langle\xi, f_{\mu}\right\rangle\right), \mu \in \Gamma\right\} \\
\text { where } \Gamma=\left\{\mu \in \Lambda(n+1, k+1) \mid \operatorname{dim}\left(F_{\mu} \cap \Pi_{\xi}^{-1}(\partial P)\right) \geq k-1\right\}
\end{gathered}
$$

(see [6, 2.6]). Hence, a tangent vector $\xi^{\prime} \in T_{\xi} \widetilde{G}(k, n)$ lies in $T_{\xi} \chi(\Phi)$ if

$$
\left\langle\xi^{\prime}, f_{\mu}\right\rangle=0
$$

whenever $\mu$ belongs to

$$
\Gamma_{0}=\left\{\mu \in \Gamma \mid\left\langle\xi, f_{\mu}\right\rangle=0\right\} .
$$

More generally, we can consider $\nu \in \Lambda(n+1, j+1), j \geq k$. Then $\left\langle\xi^{\prime}, f_{\mu}\right\rangle=0$ for all $\mu \subset \nu$ if and only if $\left.\xi^{\prime}\right\rfloor f_{\nu}=0$.

Theorem 3. Suppose

$$
\left.A=\{\xi \in \widetilde{G}(k, n) \mid \xi\rfloor f_{\mu}=0 \text { for all } \mu \in \Gamma_{0} \cup \Gamma_{1}\right\},
$$

where $\Gamma_{0} \subset \Lambda(n+1, k+1)$ and $\Gamma_{1} \subset \bigcup_{j=k+1}^{n}\{\Lambda(n+1, j+1)\}$. If $\left|\Gamma_{1}\right| \leq k-1$ and $\left|\Gamma_{1} \cup \Gamma_{0}\right| \leq n-2$, then $A$ is a union of great circles.

Proof. If $\xi^{\prime} \in T_{\xi} \widetilde{G}(k, n)$ is simple, Proposition 2 states that the great circle $C$ consisting of the unit vectors in $\operatorname{lin}\left\{\xi, \xi^{\prime}\right\}$ lies in $\widetilde{G}(k, n)$. If in addition $\xi$, 
$\xi^{\prime} \in A$, then the linearity of $\rfloor$ implies $C \subset A$. Therefore, the proof will follow if given $\xi \in A$, we can construct a simple tangent vector $\xi^{\prime} \in T_{\xi} \widetilde{G}(k, n) \cap A$.

From (2.2), we may write any simple tangent vector $\xi^{\prime} \in T_{\xi} \widetilde{G}(k, n),\left|\xi^{\prime}\right|=1$, as

$$
\left.\xi^{\prime}=t \wedge(x\rfloor \xi\right),
$$

where $x \in L(\xi)$ and $t \in L(\xi)^{\perp}$ are unit vectors. This shows that the set of simple, unit tangent vectors to $\widetilde{G}(k, n)$ at $\xi$ is isomorphic to $S^{k-1} \times S^{n-k-1}$. As will become clear below, this decomposition underlies the bounds $k-1$ and $n-2$ in the statement of the theorem.

The condition that $\xi^{\prime} \in A$ can be written as

$$
\left.\left.\left.(t \wedge(x\rfloor \xi))\rfloor f_{\mu}=t\right\rfloor((x\rfloor \xi)\right\rfloor f_{\mu}\right)=0
$$

for all $\mu \in \Gamma_{0} \cup \Gamma_{1}$ (see $\left.[4,1.5 .1]\right)$. Here we require a geometric interpretation of interior multiplication (see [6, 1.12]). If $\alpha \in \Lambda_{s} R^{n}$ and $\beta \in \Lambda_{r} R^{n}$ are simple and $r \leq s$, then $\alpha\rfloor \beta \in \Lambda_{r-s} R^{n}$ is simple with

$$
\alpha\rfloor \beta=0 \quad \text { if and only if } L(\alpha) \cap L(\beta)^{\perp} \neq\{0\},
$$

and

$$
L(\alpha\rfloor \beta)=L(\alpha)^{\perp} \cap L(\beta)
$$

otherwise. Note that if $r=s, \alpha\rfloor \beta=\langle\alpha, \beta\rangle$.

The remainder of the proof consists of dimension counting in $R^{n}$. The dimension of $L=L(\xi)$ is $\operatorname{dim} L=k$, and $\operatorname{dim}\left(L_{\mu}=L\left(f_{\mu}\right)\right)=|\mu|-1$. Equation (2.9) implies $L(\eta), \eta=x\rfloor \xi$, is a $(k-1)$-dimensional subspace of $L$.

Now since $\xi \in A, \xi\rfloor f_{\mu}=0$ for all $\mu \in \Gamma_{0} \cup \Gamma_{1}$, and therefore (2.8) gives

$$
\operatorname{dim}\left(L \cap L_{\mu}^{\perp}\right) \geq 1 \text {. }
$$

If (2.10) remains true with $L(\eta)$ in place of $L$, then again by (2.8) $\eta\rfloor f_{\mu}=$ 0 , and so $(2.7)$ holds. Thus, we choose $L(\eta)$ to contain a maximal linearly independent set of vectors from the subspaces in (2.10). Since $\operatorname{dim} L(\eta)=k-1$, (2.7) will then hold for at least $k-1$ multi-indices of $\Gamma_{0} \cup \Gamma_{1}$, including all of $\Gamma_{1}$.

Given $\eta$, we choose $t \in L^{\perp}$ by considering an index $\mu^{\prime} \in \Gamma_{0}$ not used in the definition of $\eta$, i.e., $L(\eta) \cap L_{\mu^{\prime}}^{\perp}=\{0\}$. From (2.9),

$$
\left.L(\eta\rfloor f_{\mu^{\prime}}\right)=L(\eta)^{\perp} \cap L_{\mu^{\prime}},
$$

and $\left.\operatorname{dim}(\eta\rfloor f_{\mu^{\prime}}\right)=\operatorname{dim} L_{\mu^{\prime}}-\operatorname{dim} L(\eta)=k-(k-1)=1$. Also since $\left.f_{\mu^{\prime}}\right] \xi=0$, $\operatorname{dim}\left(L^{\perp} \cap L_{\mu^{\prime}}\right) \geq 1$. However $L^{\perp} \subset L(\eta)^{\perp}$, so we must have

$$
L(\eta)^{\perp} \cap L_{\mu^{\prime}}=L^{\perp} \cap L_{\mu^{\prime}}
$$


In this case, (2.7) holds if $t$ is orthogonal to the line in (2.12). But since $\operatorname{dim} L^{\perp}=n-k$, and there are at most $\left|\Gamma_{0} \cup \Gamma_{1}\right|-(k-1) \leq n-k-1$ possibilities for $\mu^{\prime}$, we can choose $t$ orthogonal to all these lines. With this choice of $t$ and $\eta,(2.7)$ holds for all $\mu \in \Gamma_{0} \cup \Gamma_{1}$ which completes the proof.

If the indices in (2.5) used to define $\chi(\Phi)$ satisfy the conditions of Theorem 3 , then every point of $\chi(\Phi)$ lies in the relative interior of an arc $C$ of a great circle, $C \subset \chi(\Phi)$. Hence, by Proposition 1 no polytope of this type could be a minimal projection of $T^{n}$. We illustrate this method with two simple examples in $R^{3}$.

Let $P=\operatorname{conv}\left\{x_{1}, \ldots, x_{n+1}\right\}$ be a pyramid with base $F=\operatorname{conv}\left\{x_{1}, \ldots, x_{n}\right\}$ an $n$-gon. If $P=\Pi\left(T^{n}: \xi\right), \xi \in \widetilde{G}(k, n)$, then $\operatorname{dim}\left(L(\xi) \cap L_{\mu}^{\perp}\right)=1$ for $\mu=(1, \ldots, n)$. Thus $(2.8)$ gives

$$
\xi\rfloor f_{\mu}=0,
$$

and this equality, together with certain inequalities as in (2.3), suffice to determine $\chi(\Phi)$. Since $\left|\Gamma_{1}\right|=1 \leq 2$, and $\left|\Gamma_{0}\right|=0$, the conditions of Theorem 3 are satisfied and $P$ is not a minimal projection.

On the other hand, if $P=\operatorname{conv}\left\{x_{1}, \ldots, x_{8}\right\}=\Pi\left(T^{7}: \xi\right)$ is a cube, then $\left|\Gamma_{0}\right|=6$ since $P$ has 6 square faces. But $7-2=5<\left|\Gamma_{0}\right|$, so Theorem 3 does not apply. Some more detailed calculations indicate that $A$ is not a union of great circles in this case.

The next theorem shows that if $P$ is simplicial, the difficulty above does not arise.

Theorem 4. If $P=\Pi\left(T^{n}: \xi\right)$ is simplicial and $\xi$ is a local minimum for $V\left(T^{n}: \cdot\right)$, then $P$ must be a simplex with the vertices of $T^{n}$ projecting to vertices of $P$.

Proof. We show that if the conclusion does not hold, then there exists a simple tangent vector $(t \vee \eta) \in T_{\xi} \widetilde{G}(k, n)$ such that

$$
\left.t\rfloor(\eta\rfloor f_{\mu}\right)=0
$$

for all $\mu \in \Gamma_{0} \cup \Gamma_{1}$.

Let $x_{i}=\Pi\left(e_{i}: \xi\right)$, and suppose a fixed face $F_{0}$ of $P$ contains $x_{1}, \ldots, x_{m}$ in its relative interior. Let $F_{0}=\operatorname{conv}\left\{x_{\lambda_{1}}, \ldots, x_{\lambda_{j_{0}+1}}\right\}$, and let

$$
F=\operatorname{conv}\left\{x_{\lambda_{1}}, \ldots, x_{\lambda_{j+1}}\right\}
$$

be a $j$-face of $P$ containing $F_{0}$. The index $\mu=\left(1, \ldots, m, \lambda_{1}, \ldots, \lambda_{j+1}\right)$ satisfies

$$
\operatorname{dim} L_{\mu}=j+m \text { and } \operatorname{dim}\left(L \cap L_{\mu}^{\perp}\right)=k-\operatorname{dim} F=k-j .
$$

Note that $F$ must be simplicial for $\operatorname{dim} F$ to be $j$. Also,

$$
\begin{aligned}
\operatorname{dim}\left(L^{\perp} \cap L_{\mu}\right) & =\operatorname{dim} L_{\mu}-\operatorname{dim} L+\operatorname{dim}\left(L \cap L_{\mu}^{\perp}\right) \\
& =(j+m)-k+(k-j)=m .
\end{aligned}
$$


Since the last number does not depend on $j$, the equality

$$
L^{\perp} \cap L_{\mu}=L^{\perp} \cap L_{\mu_{0}}
$$

must hold for $\mu_{0}=\left(1, \ldots, m, \lambda_{1}, \ldots, \lambda_{j_{0}+1}\right)$. Hence, we may replace $\mu$ by $\mu_{0}$ in (2.14).

The method of determining $t \in L^{\perp}$ is exactly as in Theorem 3. That is, (2.11) and (2.12) give

$$
\left.L(\eta\rfloor f_{\mu_{0}}\right)=L(\eta)^{\perp} \cap L_{\mu_{0}}=L^{\perp} \cap L_{\mu_{0}},
$$

and (2.14) holds if $t$ is orthogonal to this subspace. According to (2.15), the sum of the dimensions of the spaces $L^{\perp} \cap L_{\mu_{0}}$ equals the number of points $x_{i}$ in the relative interior of faces of $P$, which is at most

$$
n+1-|\operatorname{vert} P| \leq n-k=\operatorname{dim} L^{\perp}
$$

If $P$ is not a simplex, this inequality is strict and $t$ can be chosen to give the required simple tangent vector.

So far, the choice of $\eta$ has not mattered. However, if $P$ is a simplex equality can hold in (2.17), so we must position $\eta$ to gain some freedom in determining $t$. With $x_{1}$ and $x_{\lambda_{1}}$ as above, choose $\eta$ so that

$$
L(\eta) \perp\left(x_{\lambda_{1}}-x_{1}\right) .
$$

Then by (2.8), $\eta\rfloor f_{\mu}=0$ for any index $\mu$ with $1, \lambda_{1} \in \mu$. Hence, we may exclude $x_{1}$ in the process of finding $t$. This reduces the sum on the left-hand side of (2.17) by at least one, and gives the desired result.

A polytope $P=\Pi\left(T^{n}: \xi\right)$ satisfying the conditions of Theorem 4 will be called a "weighted" simplex. It is easy to see that these polytopes are the only projections of $T^{n}$ for which the region $\chi(\Phi)$ is exactly one point.

The volume of $P$ has a nice expression in terms of its weights $w_{1}, \ldots, w_{k+1}$, $\sum w_{i}=n+1$. Consider the matrix

$$
\begin{aligned}
& \begin{array}{llll}
w_{1} & w_{2} & w_{3} & w_{k+1}
\end{array}
\end{aligned}
$$

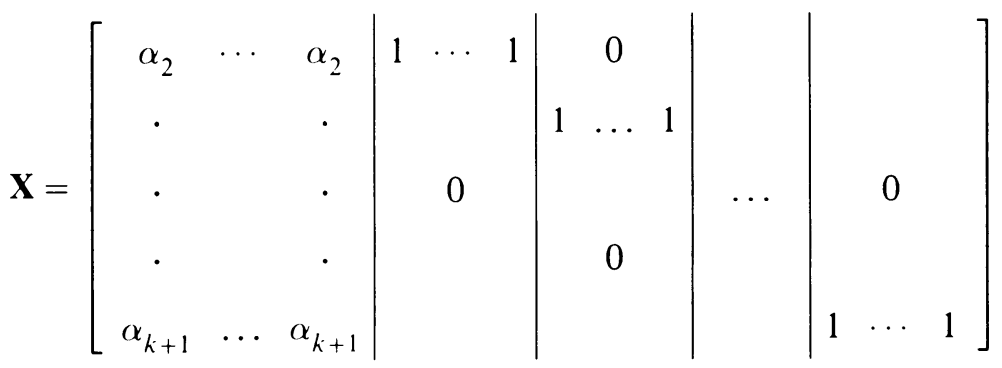


where $\alpha_{i}=-w_{i} / w_{1}$. The polytope $P^{\prime}$ which is the convex hull of the columns of $\mathbf{X}$ differs from $P$ by a linear transformation of $R^{k}$ (see $[6,4.1]$ ). Therefore, $\xi$ differs from the wedge product $\xi^{\prime}$ of the rows of $\mathbf{X}$ by a constant factor, i.e.

$$
\xi=\xi^{\prime} /\left|\xi^{\prime}\right| \text {. }
$$

Let

$$
\lambda=\left(\lambda_{1}, \ldots, \lambda_{k+1}\right) \quad \text { where } 1 \leq \lambda_{1} \leq w_{1}<\lambda_{2} \leq w_{1}+w_{2}<\lambda_{3} \leq \cdots,
$$
and let $\lambda \backslash \lambda_{i}$ be the sequence obtained from $\lambda$ by removing $\lambda_{i}$. Then

$$
\xi_{\lambda \backslash \lambda_{i}}^{\prime}=(-1)^{i+1} w_{i} / w_{1},
$$

and $\xi_{\mu}^{\prime}=0$ for all other indices $\mu \in \Lambda(n+1, k)$. Also,

$$
\left|\xi^{\prime}\right|^{2}=\sum_{\lambda(n, k)}\left(\xi_{\mu}^{\prime}\right)^{2}=\sum_{i=1}^{k+1} \frac{w_{i}^{2}}{w_{1}^{2}} \frac{\Pi w_{j}}{w_{i}}=\frac{W}{w_{1}^{2}}\left(\sum_{i=1}^{k+1} w_{i}\right)=\frac{(n+1) W}{w_{1}^{2}},
$$

where $W=w_{1} w_{2} \cdots w_{k+1}$. Substituting (2.21) and (2.22) in (2.19) gives

$$
\xi_{\lambda \backslash \lambda_{i}}=\frac{(-1)^{i+1} w_{i}}{\sqrt{(n+1) W}}
$$

The volume of $P$ is

$$
V(P)=\frac{1}{k !} \sum_{i=1}^{k+1}\left|\xi_{\lambda \backslash \lambda_{i}}\right|=\frac{1}{k !} \sqrt{\frac{n+1}{W}} .
$$

An even distribution of the weights will maximize $W$, and so minimize $V(P)$. More precisely, we write

$$
n+1=d(k+1)+r, \quad 0 \leq r<k+1,
$$

and let

$$
w_{i}= \begin{cases}d+1, & 1 \leq i \leq r \\ d, & r<i \leq k+1 .\end{cases}
$$

Conjecture A states that the simplex $T_{n}^{k}$ with the weights in (2.26) is the smallest projection of $T^{n}$ into $R^{k}$. The positive results are contained in

Theorem 5. The smallest projection of $T^{n}$ into $R^{k}$ is $T_{n}^{k}$ in the following cases:

(a) $k=1$.

(b) $k=n-1$.

(c) $k=2$.

(d) $n \leq 6$.

Proof. If $k=1$ or $n-1, \widetilde{G}(k, n)$ is isomorphic to the unit sphere $S^{n-1}$, so the minimal projection must occur when $\chi(\Phi)$ is a singleton by Lemma 1 . These points represent the weighted simplices, and the smallest is the one with even weights. The minimum width of $T^{n}$ is the distance between opposite flats 
of the middle dimensions (see also [1]). The hyperplane containing the smallest projection of $T^{n}$ is normal to an edge of $T^{n}$ (see [10, pp . 164-166]).

Case (c) is covered by Theorem 4 , since all polygons are simplicial. There remain a finite number of nonsimplicial polytopes in (d), and in each case Theorem 3 (using condition (2.13) as necessary) shows that the projection is not minimal.

The remarks about the cube preceding Theorem 4 lead us to suspect that different methods will be necessary to establish Conjecture A in higher dimensions.

Considering the minimal projections of other regular polytopes, the smallest projection of the regular $n$-cube $C^{n}$ into $R^{k}$ is $C^{k}$ (see [2, p. 108]). A theorem similar to Theorem 4 can be proved for the regular crosspolytope $X^{n}$, and using it one can show that the smallest projection is an evenly weighted crosspolytope in $R^{k}$ when $k=1$ or 2 . The higher-dimensional cases are more difficult because the singleton regions $\chi(\Phi)$ represent a wider collection of polytopes. A characterization of such polytopes is given in [11].

\section{Maximal projections}

According to Conjecture $\mathrm{B}$, the largest projection of $T^{n}$ into a $k$-dimensional subspace of $R^{n}$ should be a particular Gale transform $\bar{T}_{n}^{n-k}$ of $T_{n}^{n-k}$, the minimal projection described in (2.26). That is, if $T_{n}^{n-k}=\Pi\left(T^{n}: L\right)$, then $\bar{T}_{n}^{n-k}=\Pi\left(T^{n}: L^{\perp}\right)$. In this section we shall discuss the cases for which the conjecture is known to be true.

Let $P=\Pi\left(T^{n}: \xi\right), \xi \in \widetilde{G}(k, n)$, and let $\Phi \in \Lambda_{k} R^{n}$ be a projection form of $P$. The comass of $\Phi$ is

$$
\|\Phi\|=\max \{\langle\Phi, \xi\rangle \mid \xi \in \widetilde{G}(k, n)\},
$$

and

$$
G(\Phi)=\{\xi \in \widetilde{G}(k, n) \mid\langle\Phi, \xi\rangle=\|\Phi\|\}
$$

is the face of $\widetilde{G}(k, n)$ with outer normal $\Phi$ (see [14]). If $G(\Phi) \subset \chi(\Phi)$, then the points of $G(\Phi)$ represent maximal projections. In general, $\|\Phi\|$ gives an upper bound on the volumes of projections of $T^{n}$ with the same combinatorial type as $P$. We should mention that Theorem 10 of [6] implies a maximal projection of $T^{n}$ must be simplicial.

We begin by calculating the volume of a projection $P$ whose transform $\bar{P}=$ $\Pi\left(T^{n}: \xi\right), \xi \in \widetilde{G}(n-k, n)$, is a weighted simplex with weights $w_{1}, \ldots, w_{n-k+1}$, $\sum w_{i}=n+1$. From [6, 3.10],we find that

$$
\begin{aligned}
V(P)=\frac{\langle\Phi, \xi\rangle}{\sqrt{n+1} k !}, & \text { where } \Phi=\sum \operatorname{sign}\left(\left\langle\xi, f_{\lambda}\right\rangle\right) f_{\lambda} \text { and } \\
& f_{\lambda}=\left(e_{\lambda_{2}}-e_{\lambda_{1}}\right) \wedge \cdots \wedge\left(e_{\lambda_{n-k+1}}-e_{\lambda_{1}}\right) .
\end{aligned}
$$

The sum in (3.1) runs over those sequences $\lambda \in \Lambda(n+1, n-k+1)$ such that

$$
0 \in \operatorname{relint} \Pi\left(F_{\lambda}: \xi\right), \quad F_{\lambda}=\operatorname{conv}\left\{e_{\lambda_{1}}, \ldots, e_{\lambda_{n-k+1}}\right\} .
$$


Since $\bar{P}$ is a weighted simplex, each $\lambda$ in (2.20) represents a copy of the simplex $\bar{P}$ which contains the origin, so $f_{\lambda}$ appears in the sum in (3.1). In particular, the term $e_{\lambda \backslash \lambda_{i}}$ appears $w_{i}$ times since there are $w_{i}$ choices for $\lambda_{i}$. Hence,

$$
\Phi_{\lambda \backslash \lambda_{i}}=\left\langle\Phi, e_{\lambda \backslash \lambda_{i}}\right\rangle=\operatorname{sign}\left(\left\langle\xi, e_{\lambda \backslash \lambda_{i}}\right\rangle\right) w_{i}=(-1)^{i+1} w_{i} .
$$

Comparing (3.3) with (2.23), we see that $\Phi$ is a multiple of $\xi$. Thus $\Phi$ is simple, and its comass equals its Euclidean norm, which is attained at $\xi$. This implies that $P$ has a larger volume that any projection of $T^{n}$ with the same combinatorial type. Finally,

$$
V(P)=\frac{|\Phi|}{\sqrt{n+1} k !}=\frac{1}{\sqrt{n+1} k !}\left(\sum_{i=1}^{n} w_{i}^{2} \frac{W}{w_{i}}\right)^{1 / 2}=\frac{\sqrt{W}}{k !},
$$

where $W=w_{1} w_{2} \cdots w_{n-k+1}$. The volume in (3.4) is maximized for the even weights of (2.26), with $d$ and $r$ given by the equation

$$
n+1=d(n-k+1)+r, \quad 0 \leq r<n-k+1 .
$$

Therefore, $\bar{T}_{n}^{n-k}$ is the largest projection of $T^{n}$ into $R^{k}$ among those which have a weighted simplex as a Gale diagram.

When $n=2 k-1, T_{2 k-1}^{k-1}$ is a regular simplex with each vertex having weight 2 , and its transform $\bar{T}_{2 k-1}^{k-1}$ is the regular crosspolytope $X^{k}$. If $n>2 k-1$, $T_{n}^{n-k}$ has $k$ vertices with weight 2 , and $n+1-2 k$ vertices with weight 1 . Thus, $\bar{T}_{n}^{n-k}$ is still $X^{k}$ since the points with weight 1 do not represent vertices of the transform (see $[13,3 \mathrm{~A} 3]$ ).

We now prove

Theorem 6. The largest projection of $T^{n}$ into $R^{k}$ is $\bar{T}_{n}^{n-k}$ in the following cases:

(a) $k=1$.

(b) $k=n-1$.

(c) $k=2$.

(d) $k=n-2, n \leq 8$.

(e) $k=3$ and $n=6$.

Proof. The maximum width of $T^{n}$ is its diameter, which equals the length of an edge. The projection of $T^{n}$ into a hyperplane orthogonal to an edge is a simplex with weights $2,1, \ldots, 1$ as expected. The largest hyperplane projection is the transform of a 1-simplex with even weights (see $[10$, p. 165]). This polytope has the same type as the cyclic polytope $C(n-1, n+1)$ (see $[8,6.1 .1])$.

Cases(c) and (e), and the case $k=3$ and $n=5$ are given in [7, Tables 1 and 2], and they all match the predicted results.

The remaining cases in (d) follow from Tables 1, 2 and 3 which give the comass of the simplicial projections of $T^{d+2}$ into $T^{d}$ for $d=4,5$, and 6 . In each case, the largest comass is attained by the projection $\bar{T}_{d+2}^{d}$. 
In the remainder of this section, we shall describe the construction of Tables 1, 2 and 3. Each row will be derived from the corresponding Gale diagram in [8, p. 112]. Although the end result may seem rather meager in comparison with the amount of work involved, the techniques below seem to have promising generalizations to codimensions greater than 2 .

Suppose $P=\Pi\left(T^{n}: L\right), L \in G(n-2, n)$. Then the transform $\bar{P}=$ $\Pi\left(T^{n}: \xi\right), L(\xi)=L^{\perp}$, is 2-dimensional. If $\Phi \in \Lambda_{2} R^{n+1}$ is the form in (3.1), we let $\mathbf{M}$ be the $(n+1) \times(n+1)$ matrix whose $i j$ th entry is

$$
\Phi_{i j}=\left\langle\Phi, e_{i j}\right\rangle \text {. }
$$

The matrix $\mathbf{M}$ is skew-symmetric, and $\|\Phi\|$ is the largest norm of the eigenvalues of $\mathbf{M}$ (see $[4,1.7 .3]$ ). Thus our procedure for finding $\|\Phi\|$ is to calculate $\Phi_{i j}$, determine the characteristic polynomial $f(z)$ of $\mathbf{M}$, and then find the root of $f(z)$ with the greatest norm.

The components of $\Phi$ can be calculated using any Gale diagram isomorphic to $\bar{P}$. The most convenient diagram for us will be the contracted diagram, which is a regular $m$-gon

$$
R=\{\exp (i 2 k \pi / m) \mid k \in Z\},
$$

with $m$ odd, and vertices having weights $w_{1}, \ldots, w_{m}$ (see $\left.[8,6.3 .1]\right)$.

The definition of $\Phi$ also depends on the orientation chosen for $R^{2}$. We shall suppose that the positive direction is counterclockwise. Also, we define a positive orientation of a polygon in $R^{2}$ to be a labeling of its vertices in the counterclockwise direction about an interior point. Let $x_{1}, \ldots, x_{m}$ be such a labeling of $R$. The symbol $\left[i_{1}, \ldots, i_{r}\right]$ will denote the polygon $\operatorname{conv}\left\{x_{1}, \ldots, x_{i_{r}}\right\}$ with vertices labeled in the given order.

Now from (3.3) and (3.4), we find that $\Phi_{i j}$ counts the number of triangles $[i, j, k]$ which contain the origin. Thus if $\operatorname{sign} \Phi_{i j}>0$,

$$
\Phi_{i j}=\sum_{k=a_{i}}^{a_{j}-1} w_{k}
$$

where

$$
x_{a_{i}}=x_{i} \exp (i(\pi / 2+\pi / m)) .
$$

A very useful recurrence relation among the components of $\Phi$ follows from (3.9).

Proposition 7. For every $i, j, k \in I_{m}=\{1, \ldots, m\}$,

$$
\boldsymbol{\Phi}_{i k}= \begin{cases}\boldsymbol{\Phi}_{i j}+\boldsymbol{\Phi}_{j k}, & 0 \notin[i, j, k], \\ \boldsymbol{\Phi}_{i j}+\boldsymbol{\Phi}_{j k}-\left(\operatorname{sign} \boldsymbol{\Phi}_{i k}\right)(n+1), & 0 \in[i, j, k] .\end{cases}
$$


Proof. Suppose $0 \notin[i, j, k]$ and the terms in (3.10) all have positive sign. Then from (3.8) we obtain

$$
\boldsymbol{\Phi}_{i j}+\boldsymbol{\Phi}_{j k}=\sum_{p=a_{i}}^{a_{j}-1} w_{p}+\sum_{p=a_{j}}^{a_{k}-1} w_{p}=\sum_{p=a_{i}}^{a_{k}-1} w_{p}=\boldsymbol{\Phi}_{i k} .
$$

The results for other choices of the signs follow from (3.11) by relabeling the vertices of the triangle $[i, j, k]$.

If $0 \in[i, j, k]$, we may assume $\operatorname{sign} \Phi_{i j}=\operatorname{sign} \Phi_{j k}=1$, and $\Phi_{i k}=-1$. Then

$$
\Phi_{i k}=-\sum_{p=a_{k}}^{a_{i}-1} w_{p}=-(n+1)+\sum_{p=a_{i}}^{a_{k}-1} w_{p},
$$

since $\sum w_{p}=n+1$. Substituting this formula in (3.11) gives (3.10).

The second and longest step in finding $\|\Phi\|$ is determining the characteristic polynomial $f(z)$ of $\mathbf{M}=\left(\Phi_{i j}\right)$. There is a very nice expression for the coefficients of $f(z)$ using the $r$ th-compond matrix $\mathbf{M}^{(r)}$ of $\mathbf{M}$ (see [9, Vol. I, p. 291]). The diagonal of $\mathbf{M}^{(r)}$ consists of all $r \times r$ subminors of $\mathbf{M}$ which have the same indices for their rows and columns. Thus,

$$
f(z)=\sum(-1)^{r} a_{r} z^{n+1-r}, \quad \text { where } a_{r}=\operatorname{tr}\left(M^{(r)}\right) \text { and } a_{0}=1 .
$$

Note that $a_{r}=0$ if $r$ is odd since $M$ is skew-symmetric.

We begin with a formula for an arbitrary $2 \times 2$ minor of $\mathbf{M}$.

Proposition 8. If $a, \ldots, d \in I_{m}$ are distinct and $[a, \ldots, d]$ is positively oriented, then

$$
\left|\begin{array}{ll}
\boldsymbol{\Phi}_{a c} & \boldsymbol{\Phi}_{a d} \\
\boldsymbol{\Phi}_{b c} & \boldsymbol{\Phi}_{b d}
\end{array}\right|= \begin{cases}\boldsymbol{\Phi}_{a b} \boldsymbol{\Phi}_{c d}, & 0 \notin[a, \ldots, d] \\
\boldsymbol{\Phi}_{a b} \boldsymbol{\Phi}_{c d}+(n+1) \boldsymbol{\Phi}_{\lambda}, & 0 \in[a, \ldots, d]\end{cases}
$$

where $\lambda$ is described below.

Proof. Expanding the determinant $D$ in (3.13) gives

$$
D=\Phi_{a c} \Phi_{b d}-\Phi_{b c} \Phi_{a d} .
$$

If $0 \notin[a, \ldots, d]$, then we obtain

$$
\begin{aligned}
& \Phi_{a c}=\Phi_{a b}+\Phi_{b c}, \\
& \Phi_{b d}=\Phi_{b c}+\Phi_{c d}
\end{aligned}
$$

and

$$
\Phi_{a d}=\Phi_{a b}+\Phi_{b c}+\Phi_{c d}
$$

from (3.10). Substituting these formulae in (3.14) gives (3.13). 


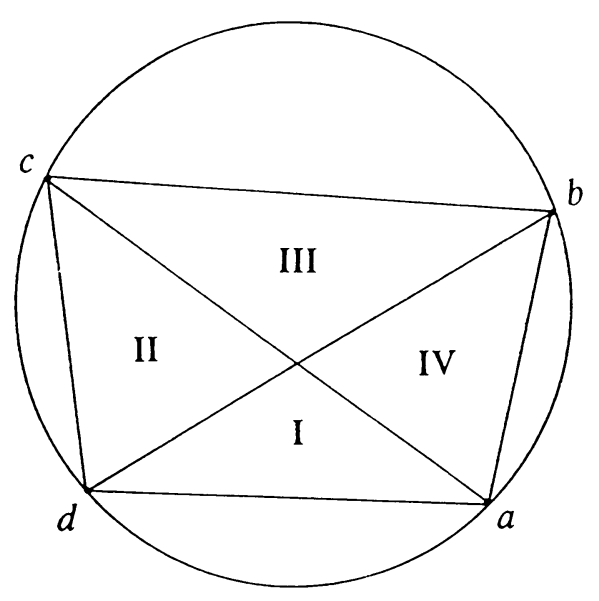

FIGURE 1

If $0 \in[a, \ldots, d]$, then the value of $D$ depends on which of the four regions pictured in Figure 1 contain the original.

I. The calculations here are exactly as above, except that (3.17) is replaced by

$$
\Phi_{a d}=\Phi_{a c}+\Phi_{c d}-(n+1)=\Phi_{a b}+\Phi_{b c}+\Phi_{a d}-(n+1),
$$

which results from two applications of (3.10). Thus

$$
D=\Phi_{a b} \Phi_{c d}+(n+1) \Phi_{b c} .
$$

II. In this case, we use (3.15), (3.18) and

$$
\Phi_{b d}=\Phi_{b c}+\Phi_{c d}-(n+1)
$$

to obtain

$$
D=\Phi_{a b} \Phi_{c d}-(n+1) \Phi_{a b} .
$$

Cases III and IV follow from I and II respectively by sending $(a, b, c, d)$ to $(c, d, a, b)$. In general, the extra term $\Phi_{\lambda}$ in (3.13) is \pm the coefficient of the side opposite the origin.

We pause here to discuss a certain function $\sigma: I_{r} \rightarrow I_{r}, r \geq 3$, whose relation to the $r \times r$ minors in (3.12) will become clear in what follows. Let $\operatorname{conv}\left\{x_{1}, \ldots, x_{r}\right\}$ be a positively oriented $r$-gon which contains the origin. Suppose also that its vertices lie on a circle centered at the origin, and that $0 \notin[i, j], i, j \in I_{r}$. Let $\theta_{i j}$ be the unique angle from $x_{i}$ to $x_{j}$ with $-\pi<\theta_{i j}<\pi$. If $i \in I_{r}$, we define $\sigma(i)$ to be the integer in $I_{r}$ with the property that

$$
0 \in[i, \sigma(i), \sigma(i)+1]
$$

$\left(x_{r+1}=x_{1}\right)$. Note that $n_{i}=\left|\sigma^{-1}(i)\right|$ can be interpreted as the weight of the edge $[i, i+1]$ in the same manner as above; i.e. $n_{i}$ counts the number of triangles $[i, i+1, k], k \in I_{r}$, which contain the origin. 
The following is a list of elementary properties of $\sigma$.

(i) $\sigma(i) \neq i, \forall i \in I_{r}$.

(ii) $\sum n_{i}=r$.

(iii) If $\theta_{i j}>0$, then $[i, j, \sigma(i), \sigma(j)]$ is positively oriented.

(iv) If $j \in \sigma^{-1}(i)$, then $[j, i, \sigma(i)]$ is a positively oriented triangle containing the origin.

(v) If $j \in \sigma^{-1}(i)$ and $[\sigma(i), k, j], k \neq \sigma(i)$, is positively oriented, then $k \in \sigma^{-1}(i)$.

(vi) $n_{i}=1$ if and only if $\sigma^{-1}(i)=\{\sigma(i)+1\}$.

(vii) $n_{i}=0$ if and only if $\sigma(i)=\sigma(i+1)$.

(viii) If $n_{i} \neq 0$, then $n_{i}=1$ if and only if $n_{\sigma(i)+1} \neq 0$.

(ix) If $\sigma$ is one-to-one, then $r$ must be odd. In this case the sequence $\left(i, \sigma(i), \ldots \sigma^{r-1}(i)\right)$ forms a star-polygon of density $\lfloor r / 2\rfloor$ (see [5, p. 107]).

The most evenly weighted case for $r$ even is described in the proposition below.

Proposition 9. Let $\sigma: I_{r} \rightarrow I_{r}$ be the mapping in (3.22) with $r \geq 4$ even, and let $r^{\prime}=r / 2$. If

(1) $n_{1}=0$,

(2) $\sigma(i)=i+r^{\prime}-1,2 \leq i \leq r^{\prime}$, and

(3) $\sigma\left(r^{\prime}+1\right) \neq r-1$,

then

(4) $\sigma(1)=r^{\prime}+1$,

(5) $\sigma\left(r^{\prime}+1\right)=r$ and

(6) $\sigma(i)=i-r^{\prime}, r^{\prime}+2 \leq i \leq r$.

Proof. Assume that (1), (2) and (3) hold. Then (1) and (vii) imply $\sigma(1)=$ $\sigma(2)=r^{\prime}+1$, which gives (4). Also by (iii), $\left[r^{\prime}, r^{\prime}+1, r-1, \sigma\left(r^{\prime}+1\right)\right]$ is positively oriented, and by (iv), $\left[1, r^{\prime}+1, \sigma\left(r^{\prime}+1\right)\right]$ is a positiveiy oriented triangle. Since $\sigma\left(r^{\prime}+1\right) \neq r-1$, the only possibility for $\sigma\left(r^{\prime}+1\right)$ is $r$.

In order to prove (6), let $r^{\prime}+2 \leq i \leq r$. From (iii), $\left[i, 1, \sigma(i), r^{\prime}+1\right]$ is positively oriented. But $\sigma(i) \neq 1$, since $n_{1}=0$, and $\sigma(i) \neq r^{\prime}+1$, since otherwise $\left[i, r^{\prime}+1, r\right]$ would be a positively oriented triangle contradicting our choice of $i$. Altogether, we find that

$$
2 \leq \sigma(i) \leq r^{\prime} .
$$

Substituting $\sigma(i)$ for $i$ in (vii), and applying (2), we obtain $n_{\sigma(i)} \neq 0$. Therefore by (viii), $n_{\sigma(i)}=1$. Replacing $i$ by $\sigma(i)$ in (vi) and (2), we thus obtain

$$
i=\sigma^{2}(i)+1=\sigma(i)+r^{\prime}-1+1,
$$

which proves $(6)$.

It should be mentioned that a similar proof as above can be used to show that $\sigma$ satisfies (1)-(6) if and only if $n_{1}=0, n_{r^{\prime}+1}=2$ and $n_{i}=1$ otherwise.

We are now ready to prove the following theorem. 
Theorem 10. Let $\left[i_{1}, \ldots, i_{r}\right]$ be a positively oriented $r$-gon, $r \geq 4$ even, whose vertices belong to $R$ (see (3.7)), and let $\sigma: I_{r} \rightarrow I_{r}$ be defined as in (3.22) using this $r$-gon. Then the $r \times r$ minor $\mathbf{X}$ of $\mathbf{M}$ with $i_{1}, \ldots, i_{r}$ as row and column indices has a nonzero determinant if and only if $\sigma$ equals the function given by (1)-(6) of Proposition 9. In this case the determinant of $\mathbf{X}$ is

$$
|\mathbf{X}|=(n+1)^{r-2} \Phi_{i_{1} i_{2}}^{2}
$$

where $n_{i_{1}}=0$.

Proof. In order to simplify our notation, we shall relabel the vertices of $R$ so that $\left(i_{1}, \ldots, i_{r}\right)=(1, \ldots, r)$. Also, we may assume $n_{1}=0$, since $\sigma$ cannot be injective (see (ix)).

The matrix $\mathbf{X}$ can be written as

$$
\mathbf{X}=\left[\begin{array}{cc|ccc}
0 & \Phi_{12} & \Phi_{13} & \cdots & \Phi_{1 r} \\
-\Phi_{12} & 0 & \Phi_{23} & \cdots & \Phi_{2 r} \\
\hline-\Phi_{13} & -\Phi_{23} & & & \\
\vdots & \vdots & & * & \\
-\Phi_{1 r} & -\Phi_{2 r} & & &
\end{array}\right]
$$

Performing elementary row operations on $\mathbf{X}$, we obtain an equivalent matrix

$$
\mathbf{X}^{\prime}=\left[\begin{array}{ccc|ccc}
0 & & \Phi_{12} & \Phi_{13} & \cdots & \Phi_{1 r} \\
-\Phi_{12} & & 0 & \Phi_{23} & \ldots & \Phi_{2 r} \\
& & & & & \\
& 0 & & & \mathbf{Y} &
\end{array}\right] .
$$

where the $i j$ th-element of $\mathbf{Y}$ is

$$
\mathbf{Y}_{i j}=\Phi_{i j}-\frac{1}{\Phi_{12}}\left|\begin{array}{ll}
\Phi_{1 i} & \Phi_{i j} \\
\Phi_{2 i} & \Phi_{2 j}
\end{array}\right| \text {. }
$$

Note that $\mathbf{Y}$ is skew-symmetric. Also, our operations have not changed $|\mathbf{X}|$, and therefore

$$
|\mathbf{X}|=\Phi_{12}^{2}|\mathbf{Y}| \text {. }
$$

The determinant $D_{i j}$ in (3.26) can be calculated from (3.10). If $0 \notin$ $[1,2, i, j]$, then $D_{i j}=\Phi_{12} \Phi_{i j}$ and $\mathbf{Y}_{i j}=0$. If $0 \in[1,2, i, j]$, then $\mathbf{Y}_{i j}=$ $\pm(n+1) \Phi_{\lambda} / \Phi_{12}$, where $\lambda$ depends on which of the four regions in Figure 1 contains the origin. The main value of $\sigma$ is that it pins down the location of the origin in this figure.

The key index is $\sigma(1)$, which equals $\sigma(2)$ by (vii) and our assumption that $n_{1}=0$. It follows from (3.10) that if $3 \leq i \leq \sigma(1)$, then

$$
\mathbf{Y}_{i j}= \begin{cases}n+1, & \sigma(1)<j \leq \sigma(i) \\ 0, & \text { otherwise }\end{cases}
$$


We also find that $\mathbf{Y}_{i j}=0$ if $\sigma(1)<i, j \leq r$. These results imply $\mathbf{Y}$ can be written as

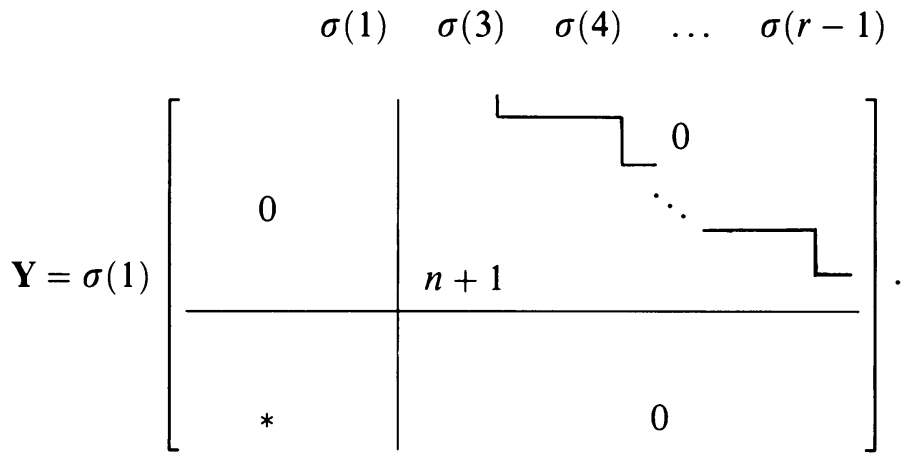

If $|\mathbf{Y}| \neq 0$, then the square blocks of zeros must have the same size, which implies $\sigma(1)=r^{\prime}+1$. Also, (2) and (3) of Proposition 9 must hold so that the columns of $\mathbf{Y}$ are distinct. Finally, since $\mathbf{Y}$ is skew-symmetric, (3.25) follows from (3.29) and (3.27).

This theorem will enable us to simplify the expression for the characteristic polynomial $f(z)$ in (3.12). Let the regular $m$-gon $R$ in (3.7) have its original counterclockwise labeling $x_{1}, \ldots, x_{m}$ and weights $w_{1}, \ldots, w_{m}$. Also, let $A_{r} \subset$ $\Lambda(n+1, r)$ be the collection of sequences $\left(i_{1}, \ldots, i_{r}\right)$ such that the function $\sigma$ defined on $\left[i_{1}, \ldots, i_{r}\right]$ satisfies (1)-(6) of Proposition 9. Then (3.25) and (3.12) imply

$$
a_{r}=(n+1)^{r-2} \sum_{A_{r}} \Phi_{i_{1} i_{2}}^{2} w_{i_{1}}, \ldots, w_{i_{r}} .
$$

In order to obtain an expression in the weights alone, we use (3.8) to write

$$
\Phi_{i_{1} i_{2}}^{2}=\sum w_{j_{1}} w_{j_{2}}, \quad \text { where } 0 \in\left[j_{k}, i_{1}, i_{2}\right], k \in\{1,2\} .
$$

If $\theta_{j_{1} j_{2}}>0$, then the function $\sigma$ defined on the $(r+2)$-gon $\left[i_{1}, \ldots, i_{r^{\prime}+1}, j_{1}\right.$, $\left.j_{2}, i_{r^{\prime}+2}, \ldots, i_{r}\right]$ has weights $n_{j_{1}}=0, n_{i_{1}}=2$ and $n_{i}=1$ otherwise. Therefore the remark following Proposition 9 implies that this sequence belongs to $A_{r+2}$. We denote the sequences in which $j_{1}=j_{2}$ by $\hat{A}_{r+2}$. Note that a sequence in $A_{r+2}$ or $\hat{A}_{r+2}$ can arise from only one term in (3.30). Thus (3.30) can be written as

$$
a_{r}=(n+1)^{r-2}\left(2 \sum_{A_{r+2}} w_{I}+\sum_{\hat{A}_{r+2}} w_{I}\right),
$$

where $w_{I}=w_{i_{1}} w_{i_{2}} \cdots w_{i_{r+2}}$.

Now let $S_{r+1}$ be the collection of sequences for which $\sigma$ is one-to-one; i.e. $\left[i_{1}, \sigma\left(i_{1}\right), \ldots, \sigma^{r}\left(i_{1}\right)\right]$ is a star-polygon of density $\lfloor(r+1) / 2\rfloor$ (see (ix)). Adding 
a vertex of $R$ to such a polygon creates a pair of opposite edges with weights 0 and 2, and thus gives a sequence in $A_{r+2}$ or $\hat{A}_{r+2}$. On the other hand, we may drop either vertex of the 0 edge of a sequence in $A_{r+2}$ to obtain one in $S_{r+1}$. The sequences in $\hat{A}_{r+2}$ are just star-polygons with one doubled vertex. Altogether, we obtain

$$
a_{r}=(n+1)^{r-1} \sum_{S_{r+1}} w_{I} .
$$

In fact, the same argument as above shows that this formula also holds when $r=2$.

Now, the coefficients in (3.12) are 0 if $r$ is odd or $r \geq m$. Hence, we may reduce $f(z)$ to

$$
\sum_{k=0}^{m^{\prime}} a_{2 k} z^{m-1-2 k}
$$

where $m^{\prime}=\lfloor m / 2\rfloor$. Substituting $z^{2}=(n+1)^{2} x$ and (3.33) in (3.34), and dividing by $(n+1)^{m-2}$, gives the polynomial

$$
g(x)=\sum_{k=0}^{m^{\prime}} s_{2 k+1} x^{m^{\prime}-k}, \quad \text { where } s_{r}=\sum_{s_{r}} w_{I} \text { and } s_{1}=n+1 .
$$

This completes our simplification of the characteristic polynomial of $\mathbf{M}$.

The final step is to find the root $x_{0}$ of (3.35) with the greatest magnitude. Then

$$
\|\Phi\|=(n+1) \sqrt{\left|x_{0}\right|}
$$

and by $(3.1)$

$$
V(P)=\frac{\sqrt{(n+1)\left|x_{0}\right|}}{k !} .
$$

Comparing (3.37) with (3.4), we see that when $k=n-2$ Conjecture $\mathrm{B}$ is equivalent to

$$
\left|x_{0}\right| \leq W /(n+1),
$$

with equality if and only if $P=\bar{T}_{2}^{n}$. In case $6 \leq n \leq 8$, the roots of $g(x)$ in (3.35) are easily calculated, and the results appear in Tables 1,2 and 3 (to within $10^{-3}$ ). The corresponding Gale diagrams are pictured in [8, p. 112].

We conclude with some remarks on the case when all the weights $w_{i}$ equal 1 , and $m=n+1$ is odd. A fairly simple counting argument shows that the coefficients of $g(x)$ in (3.35) are then

$$
s_{r}=\left|S_{r}\right|=\frac{n+1}{r}\left(\begin{array}{c}
n^{\prime}+r^{\prime} \\
r-1
\end{array}\right) \text {. }
$$


TABLE $1 . d=4$.

$\begin{array}{ccccc} & s_{3} & s_{5} & s_{7} & \text { comass }(\div 1 / \sqrt{7}) \\ 1 & 12 & & & 3.464 \\ 2 & 11 & 3 & & 2.923 \\ 3 & 12 & 4 & & 2.971 \\ 4 & 13 & 4 & & 3.205 \\ 5 & 14 & 7 & 1 & 3.049\end{array}$

TABLE 2. $d=5$.

$\begin{array}{rrrrc} & s_{3} & s_{5} & s_{7} & \operatorname{comass}(\div 1 / \sqrt{8}) \\ 1 & 16 & & 4 \\ 2 & 18 & & 4.243 \\ 3 & 14 & 4 & 3.335 \\ 4 & 16 & 6 & 3.464 \\ 5 & 18 & 6 & 3.84 \\ 6 & 18 & 8 & 3.623 \\ 7 & 20 & 8 & & 4 \\ 8 & 20 & 12 & 2 & 3.696\end{array}$

However, $\|\Phi\|$ can more easily be calculated from the matrix $\mathbf{M}=\left(\Phi_{i j}\right)$. The first row of $\mathbf{M}$ is

$$
\left(0,1,2, \ldots, n^{\prime},-n^{\prime},-n^{\prime}+1, \ldots,-1\right)
$$

and the $i$ th row is obtained by shifting (3.40) $i$ places to the right. Because of the cyclic structure of $\mathbf{M}$, its eigenvectors $y_{k}$ are composed of roots of unity; i.e.

$$
\begin{aligned}
\text { (3.41) } y_{k}=\left(z_{k}, z_{k}^{2}, \ldots, z_{k}^{n}\right), & \text { where } z_{k}=\exp (i k \theta), k \in\{0, \ldots, n\} \\
& \text { and } \theta=2 \pi /(n+1) .
\end{aligned}
$$

The regular $(n+1)$-gon occurs when $k=1$, and the corresponding eigenvalue is

$$
2 i \sum_{j=1}^{n^{\prime}} j \sin (j \theta) .
$$


TABLE 3. $d=6$.

\begin{tabular}{|c|c|c|c|c|}
\hline & $s_{3}$ & $s_{5}$ & $s_{7}$ & $\operatorname{comass}(\div 1 / 3$ \\
\hline 1 & 20 & & & 4.472 \\
\hline 2 & 24 & & & 4.9 \\
\hline 3 & 27 & & & 5.196 \\
\hline 4 & 17 & 5 & & 3.704 \\
\hline 5 & 20 & 8 & & 3.91 \\
\hline 6 & 23 & 8 & & 4.389 \\
\hline 7 & 21 & 9 & & 3.989 \\
\hline 8 & 25 & 9 & & 4.602 \\
\hline 9 & 24 & 12 & & 4.243 \\
\hline 10 & 27 & 12 & & 4.703 \\
\hline 11 & 28 & 12 & & 4.836 \\
\hline 12 & 23 & 12 & & 4.053 \\
\hline 13 & 28 & 16 & & 4.606 \\
\hline 14 & 26 & 17 & 3 & 4.29 \\
\hline 15 & 27 & 20 & 4 & 4.243 \\
\hline 16 & 28 & 20 & 4 & 4.44 \\
\hline 17 & 29 & 21 & 4 & 4.54 \\
\hline 18 & 30 & 27 & 9 & 4.385 \\
\hline
\end{tabular}

This formula was used to calculate the comass in Table 1, row 5, and Table 3, row 18. With more sophisticated combinatorial arguments, it may be possible to establish (3.38) for diagrams with arbitrary weights.

\section{REFERENCES}

1. R. Alexander, The width and diameter of a simplex, Geom. Dedicata 6 (1977), 87-94.

2. G. D. Chakerian and P. Filliman, The measures of the projection of a cube, Studia Sci. Math. Hungar. 21 (1986), 103-110.

3. H. S. M. Coxeter, Regular polytopes, Dover, New York, 1973.

4. H. Federer, Geometric measure theory, Springer-Verlag, 1969.

5. L. Fejes Tòth, Regular figures, Macmillan, 1964. 
6. P. Filliman, Exterior algebra and projections of polytopes, Geom. Dedicata (to appear).

7. _ The largest projections of regular polytopes, Isreal J. Math. 64 (1988), 207-228.

8. B. Grünbaum, Convex polytopes, Wiley, 1967.

9. W. Hodge and D. Pedoe, Methods of algebraic geometry, Cambridge Univ. Press, 1947.

10. H. Martini and B. Weissbach, Zur besten Beleuchtung konvexer Polyeder, Beiträge Algebra Geom. 17 (1984), 151-168.

11. P. McMullen, Linearly stable polytopes, Canad. J. Math. 21 (1969), 1427-1431.

12. P. McMullen and G. C. Shephard, Convex polytopes and the upper bound conjecture, London Math. Soc. Lecture Note Ser., no. 3, Cambridge Univ. Press, 1971.

13. P. McMullen, Transforms, diagrams and representations, Contributions to Geometry (Proc. Geometry-Sympos., Siegen), J. Tolke and J. M. Wills, editors, Birkhäuser, 1978.

14. F. Morgan, The exterior algebra $\Lambda^{k} R^{n}$ and area minimization, Linear Algebra Appl. 66 (1985), 1-28.

Department of Mathematics, Western Washington University, Bellingham, WashingTON 98225

Current address: Department of Mathematics, White Hall, Cornell University, Ithaca, New York 14853 